Páginas: 13-28-

Recibido: 2020-03-04

Revisado: 2020-07-03

Aceptado: 2020-07-27

Preprint: 2020-09-01

Publicación Final: 2021-01-31 www.revistascientificas.us.es/index.php/fuentes/index

DOI: https://doi.org/10.12795/revistafuentes.2021.v23.i1.11383

\title{
Autorrevelación docente: una revisión de la literatura
}

\author{
Teacher Self-Disclosure: a literature review
}

\author{
iD Facundo Alberto Peña Froment \\ Universidad de Sevilla (España) \\ iD Alfonso Javier García González \\ Universidad de Sevilla (España) \\ D María Rocío Bohórquez Gómez-Millán \\ Universidad de Sevilla (España)
}

\section{Resumen}

Para maximizar el aprendizaje de los estudiantes, los docentes exhiben intencionalmente determinados comportamientos para construir relaciones interpersonales con el alumnado. Una de las conductas que los docentes incorporan en su docencia para clarificar y mejorar la comprensión de los estudiantes sobre el contenido del curso es la autorrevelación. Sin embargo, cuando se emplea de forma inadecuada, ésta puede perjudicar las oportunidades de aprendizaje de los estudiantes. De este modo, el objetivo de esta investigación consistió en determinar el estado del arte sobre la autorrevelación docente mediante una revisión sistemática de la literatura existente, estableciendo su efecto en el proceso de enseñanza-aprendizaje. Se llevó a cabo una búsqueda bibliográfica en diferentes bases de datos nacionales e internacionales, seleccionándose 39 artículos científicos para la revisión, publicados entre 1982 y 2019 , que respetaron los criterios de inclusión fijados. Con respecto al efecto de la autorrevelación docente en el proceso de enseñanzaaprendizaje, se establecieron 6 categorías: aprendizaje cognitivo, aprendizaje afectivo, motivación académica, participación e interés, comunicación profesor-alumno fuera del aula y percepciones y evaluaciones sobre el docente, incorporándose en cada una de ellas los principales resultados de los artículos incluidos en el análisis. Atendiendo a los hallazgos obtenidos, se recalca la necesidad de que, para que favorezca el aprendizaje de los estudiantes, las autorrevelaciones de los docentes deben ser moderadas, relevantes y positivas.

\begin{abstract}
To maximize student learning, teachers intentionally exhibit certain behaviors to build interpersonal relationships with students. One of the behaviors that teachers incorporate in their teaching to clarify and improve students' understanding of the course content is self-disclosure. However, when it is used inappropriately, it may damage student learning opportunities. Thus, the purpose of this research was to establish the state of the art on teacher self-disclosure through a systematic review of the existing literature, establishing itseffect on the teaching-learning process. A bibliographic search was carried out in different national and international databases, selecting 39 scientific articles for the revision, published between 1982 and 2019, that met the established inclusion criteria. Regarding the effect of teacher self-disclosure on the teaching-learning process, 6 categories weredetermined: cognitive learning, affective learning, academic motivation, participation and interest, teacher-student out-of-class communication and perceptions and evaluations of the teacher, including in each of them the main results of the articles included in the analysis. Based on the obtained results, it is highlighted the need that, in order to promote student learning, teacher self-disclosure must be moderate, relevant and positive.
\end{abstract}

\section{Palabras clave / Keywords}

Revisión de literatura, Autorrevelación docente, Comportamiento del docente, Relación profesor-alumno, Proceso de enseñanzaaprendizaje.

Literature Review, Teacher self-disclosure, Teacher behavior, Teacher-Student relationship, Teaching-Learning process. 


\section{Introducción}

En el aula los docentes pasan más tiempo hablando que el alumnado (McBride y Wahl, 2005), comunicándose con los estudiantes para desarrollar relaciones interpersonales (Frymier y Houser, 2000). Aunque la mayor parte del tiempo la dedican para abordar el contenido del curso, también hablan sobre ellos mismos, contando historias o compartiendo creencias personales (Nussbaum, Comadena y Holladay, 1987). Así, la clase supone un espacio en donde el profesorado comparte cuestiones del ámbito personal o privado con el alumnado (Goodboy et al., 2014). Lo que comparten y la forma en la que lo hacen se denomina la autorrevelación del docente (en inglés, Teacher/Instructor Self-Disclosure) (McCarthy y Schmeck, 1982), constituyendo una estrategia empleada por parte del profesorado para establecer relaciones satisfactorias y positivas con los estudiantes (Cooper y Simonds, 1999). La autorrevelación docente puede definirse como el intercambio de información de carácter personal y/o profesional de un docente sobre sí mismo de una forma creíble (Cozby, 1973). Nussbaum y Scott (1979) indicaron que la autorrevelación docente supone compartir con los estudiantes información personal de manera intencional o no intencional. De acuerdo a Sorensen (1989), la autorrevelación docente trata de afirmaciones sobre el docente que pueden tener o no relación con el contenido de las asignaturas impartidas, pero revelan información sobre el docente que difícilmente puedan obtener los estudiantes de otras fuentes. Finalmente, Goldstein y Benassi (1994) definieron la autorrevelación docente como el intercambio de información personal y profesional del profesorado con el alumnado, siendo ésta la definición más aceptada.

Javidi y Long (1989) identificaron temas comunes sobre la autorrevelación de los docentes, incluyendo su educación y experiencia docente, familias, amigos y colegas, creencias y opiniones y actividades de ocio. De manera similar, McBride y Wahl (2005) hallaron que el profesorado reveló información privada sobre sus experiencias de vida, relaciones familiares, actividades cotidianas y emociones, pero no compartió información sus relaciones negativas, actividades sexuales, salarios u otro tipo de información que pudiera perjudicar su credibilidad. Asimismo, Sumuer, Esfer y Yildirim (2014) afirman que los docentes publican en Facebook su nivel educativo, lugar de nacimiento, sexo, nombres, fecha de nacimiento y residencia y evitan publicar sus tendencias políticas, correos electrónicos, series o programas preferidos y creencias religiosas. Siguiendo estas líneas, DiVerniero y Hosek (2011) revelan que el alumnado percibió las relaciones familiares y los antecedentes y la experiencia docente como formas apropiadas de autorrevelación tanto dentro como fuera del aula, mientras que percibieron los problemas personales y las opiniones políticas y religiosas de los docentes como autorrevelaciones inapropiadas. De manera similar, el alumnado percibe que la autorrevelación de los docentes sobre relaciones familiares es menos apropiada que la divulgación que se encuentra relacionada con el contenido de clase (Paluckaite y Zardeckaite-Matulaitiene, 2015). Hosek y Presley (2018) hallaron que las relaciones familiares, los antecedentes y las experiencias docentes fueron considerados por los estudiantes como formas apropiadas de autorrevelación del docente, mientras que, por el contrario, compartir relaciones sentimentales o íntimas, problemas personales, creencias y opiniones políticas y religiosas y consumo de alcohol se consideraron temas inapropiados.

Zhang, Shi, Tonelson y Robinson (2009) afirman que el profesorado tiene actitudes negativas hacia la divulgación de sus creencias religiosas y perspectivas políticas y de información sobre sus relaciones sentimentales. Del mismo modo, Zhang (2010) señala que los docentes están de acuerdo en evitar compartir con los estudiantes sus creencias y prácticas religiosas y/o políticas por temor a estar en desacuerdo con el alumnado, por la posibilidad de que éste no comprenda o malinterprete dicha información y por el riesgo de que surjan conflictos con los padres y la administración educativa. En líneas similares, Rahimi y Bigdeli (2016) apuntan que el profesorado considera apropiadas las autorrevelaciones sobre experiencias personales, familia y vida social mientras que las autorrevelaciones relacionadas con creencias políticas y religiosas son consideradas inapropiadas.

Asimismo, los propósitos o intenciones que el colectivo docente atribuye a sus autorrevelaciones varían. Downs, Javidi y Nussbaum (1988) indican que cuando el profesorado comparte con los estudiantes cuestiones del ámbito privado, lo realiza para debatir sobre una determinada temática o para aclarar el contenido que se imparte. De acuerdo a Hosek y Thompson (2009), el profesorado comparte información de carácter personal acercarse al alumnado y para explicar el contenido de la asignatura impartida. De modo similar, Webb (2014) señala que la autorrevelación se emplea para establecer relaciones interpersonales con los estudiantes, para aumentar su credibilidad, para explicar el contenido de las asignaturas y también para mantener la atención del alumnado.

Zhang et al. (2009) examinaron las opiniones del colectivo docente sobre los motivos por los que los docentes comparten información personal en el aula, descubriendo que el profesorado considera que crear un ambiente 
de clase positivo, aclarar el contenido de clase, establecer modelos sociales, mejorar los intereses de los estudiantes, crear relaciones positivas con el alumnado y atraer la atención de los estudiantes son razones adecuadas de autorrevelación; en cambio, entretener al alumnado y complacer a los propios docentes se contemplaron como intenciones inapropiadas. En relación al alumnado, éste interpreta las revelaciones por parte del profesorado de información privada como intentos de establecer conexiones personales, de fomentar un ambiente de clase positivo y abierto y de ser honestos con ellos (Cayanus, Martin y Goodboy, 2009). Igualmente, los estudiantes aprecian las autorrevelaciones de los docentes ya que consideran que minimizan las diferencias de estatus entre el profesorado y el alumnado (Myers, Brann y Members of Comm 600, 2009). Cayanus y Martin (2008) identificaron tres factores de la autorrevelación docente: cantidad, relevancia y negatividad. La cantidad supone con qué frecuencia y cuánta información personal comparte el docente con los estudiantes; la relevancia hace referencia a si la información personal que revela el docente está relacionada con el contenido de clase y la negatividad se refiere a informaciones inadecuadas, inapropiadas o negativas del ámbito privado del docente. Atendiendo a la cantidad de autorrevelaciones llevadas a cabo por los docentes, Downs et al. (1988) señalan que el alumnado prefiere en la clase cantidades moderadas de autorrevelación. Del mismo modo, Myers et al. (2009) indican que el alumnado espera que los docentes no revelen información personal en exceso, sino que equiparen la cantidad de autorrevelación con el contenido de las asignaturas. Así, Baker, Clark-Gordon y Myers (2019) sugieren que los docentes consideren no sólo la cantidad sino también la frecuencia con la que comparten información privada con el alumnado ya que, de lo contrario, corren el riesgo de perjudicar su aprendizaje.

Con respecto a la relevancia de las autorrevelaciones, ésta es considerada como la más importante para los estudiantes ya que, por un lado, transmite información del curso de una manera que los estudiantes aprecian $y$, por otro lado, refuerza la comprensión del material al conectarlo con experiencias reales fuera de clase (Schrodt, 2013). Lannutti y Strauman (2006) postulan que el alumnado espera que el profesorado divulgue información privada que sea relevante para el contenido de las asignaturas que se impartan ya que, si no se encuentra relacionada con lo que se aborda en el aula, puede resultar inapropiada o fuera de lugar en la clase. Como concluyen Miller, Katt, Brown y Sivo (2014), mantener las autorrevelaciones de los docentes relevantes para el aprendizaje de los estudiantes es de suma importancia si se desean utilizar como comportamientos de enseñanza efectivos.

En cuanto a la negatividad de las autorrevelaciones, ésta puede considerarse negativa cuando es ofensiva, demasiado íntima o irrelevante para el contenido del curso (Frisby y Sidelinger, 2013). En esta línea, la divulgación de información personal por parte de los docentes sobre política, religión o problemas personales es percibida por los estudiantes como inapropiada (Hosek y Thompson, 2009). Según Kearney, Plax, Hays e Ivey (1991), las autorrevelaciones docentes de carácter negativo pueden ser perjudiciales para el clima del aula y generar evaluaciones desfavorables de los estudiantes sobre el colectivo docente. En este sentido, Dalto, Ajzen y Kaplan (1979) descubrieron que sujetos que compartieron información personal negativa fueron percibidos más desfavorables que quienes revelaron información personal positiva. Como apuntan Coffelt, Strayhorn y Tilson (2014), una autorrevelación positiva podría generar un efecto positivo en el aprendizaje del alumnado, del mismo modo que las percepciones de revelaciones inadecuadas podrían tener un efecto negativo en el aprendizaje del alumnado.

En síntesis, la autorrevelación docente es una estrategia que los docentes pueden incorporar en su docencia para enriquecer las relaciones entre el profesorado y el alumnado (Zardeckaite-Matulaitiene y Paluckaite, 2013). Como indica Deiro (2005), la autorrevelación no solo permite desarrollar una relación respetuosa y afectiva entre docentes y estudiantes, sino también puede contribuir al desarrollo de un clima positivo del aula y a la mejora del aprendizaje del alumnado. Por esta razón, Buskist y Saville (2004) recomiendan al colectivo docente que comparta con el alumnado una parte de sí mismos. Sin embargo, la información personal que se debe revelar al alumnado supone un dilema para los docentes debido fundamentalmente a que desconocen cómo reaccionarían los estudiantes y cómo se vería afectado el clima del aula (Meluch, Feehan y Starcher, 2019). Así, el principal objetivo de esta investigación consiste en determinar el estado del arte sobre la autorrevelación docente mediante una revisión sistemática de la literatura, estableciendo su efecto en el proceso de enseñanza-aprendizaje.

\section{Metodología}

Para responder al objetivo establecido, se realizó una búsqueda de estudios sobre autorrevelación docente en distintas bases de datos nacionales e internacionales para seleccionar los artículos objeto de análisis. Para la búsqueda se utilizaron las bases de datos Web of Science, Scopus, ERIC y PsycINFO en inglés y las 
propias del CSIC y Dialnet en español. Como criterio de búsqueda de los artículos se estableció la identificación en el título o resumen de los artículos de las palabras clave Teacher SelfDisclosure/Autorrevelación docente e Instructor Self-Disclosure/Autorrevelación del profesor, obtenidas del tesauro de ERIC. Igualmente, con el propósito de acotar la búsqueda, estas palabras clave se combinaron con las tres dimensiones de la autorrevelación docente identificadas por Cayanus y Martin (2008): Amount/Cantidad, Relevance/Relevancia y Negativity/Negatividad. Los artículos hallados fueron sometidos a un procedimiento de selección en el que se compararon con distintos criterios para incluirlos en la revisión. Se estableció como criterio estándar el análisis de cualquier artículo científico que proporcionara datos sobre el efecto de las autorrevelaciones del profesorado en el proceso de enseñanza-aprendizaje. Asimismo, se establecieron los siguientes criterios de inclusión:

- Tipo de documento: se incluyeron únicamente artículos científicos, de manera que se excluyeron tesis doctorales, comunicaciones de congresos, tesinas, libros y capítulos de libros.

- Tipo de artículo: se seleccionaron los de carácter empírico, de modo que se excluyeron los metaanálisis, los estudios psicométricos y las revisiones teóricas.

- Carácter de la variable de autorrevelación docente: se incluyeron investigaciones que abordaron la autorrevelacióndocente como variable independiente, excluyéndose estudios que analicen la autorrevelación docente como variable dependiente o como variable mediadora.

Del conjunto de documentos hallados ( $\mathrm{N}=111), 39$ artículos respetaban los criterios de inclusión fijados, el resto de artículos se excluyeron de la revisión de la literatura (ver Anexo 1). Los artículos escogidos para el análisis se sitúan entre los años 1982 y 2019, ambos inclusive. Asimismo, cabe indicar que los artículos seleccionados par el análisis están escritos en inglés, no habiéndose hallado investigaciones en otros idiomas. Los artículos escogidos se examinaron de acuerdo al efecto de las autorrevelaciones del profesorado en el proceso de enseñanza-aprendizaje.

Para crear las categorías o temas de análisis propios de los datos descriptivos, los investigadores siguieron las indicaciones de Braun y Clarke (2006) y Guest, MacQueen y Namey (2012), quienes establecen un proceso inductivo mediante el cual se lleva a cabo un análisis exhaustivo del contenido de las investigaciones seleccionadas por medio de un análisis temático.De esta forma, se elaboraron códigos iniciales atendiendo al contenido semántico -explícito- de los estudios, agrupándose posteriormente en temas. Con el propósito de garantizar la validez del proceso de identificación de temas, se creó un glosario de los mismos y se realizó la codificación de forma paralela e independiente por parte de dos investigadores en oleadas sucesivas hasta lograr un acuerdo total en la codificación; de esta manera, se comenzó con un Coeficiente Kappa de Cohen $=.70$, hasta alcanzar el valor $\mathrm{K}=1$.

\section{Resultados}

Se determinó el efecto de las autorrevelaciones del profesorado en el proceso de enseñanza-aprendizaje tras analizar los artículos objeto de estudio, identificándose 6 categorías: aprendizaje cognitivo, aprendizaje afectivo, motivación académica, participación e interés, comunicación profesor-alumno fuera del aula y percepciones y evaluaciones sobre el docente.

\subsection{Aprendizaje cognitivo}

El aprendizaje cognitivo consiste en la capacidad de un individuo para retener y comprender información (Edwards, Edwards, Shaver y Oaks, 2009). McCarthy y Schmeck (1982) hallaron que la autorrevelación del docente se asoció positivamente con una mayor retención del contenido de clase mientras que Gnanaselvam y Kepol (2018) hallaron que la cantidad y la relevancia de la autorrevelación se asoció positivamente con la mejoría en la escritura de los estudiantes, sugiriendo con ello que la autorrevelación docente contribuye al fucnionamiento cognitivo del alumnado.

\subsection{Aprendizaje afectivo}

El aprendizaje afectivo se define como la actitud positiva del estudiante ante la asignatura y el docente (Bloom, 1976). Diversos estudios señalan que la autorrevelación docente se relaciona positivamente con el 
aprendizaje afectivo (Cayanus y Martin, 2008; Goodboy et al., 2014; Jebbour, 2018; Sorensen, 1989), aunque algún estudio (Cayanus y Martin, 2004) no pudo hallar dicha relación.

Mazer, Murphy y Simonds (2007) analizaron la influencia de la autorrevelación docente en Facebook sobre el clima del aula y el aprendizaje afectivo del alumnado, concluyendo que los estudiantes que observaron el perfil de un profesor que contenía mucha información personal percibieron un clima más positivo en el aula y mostraron mayor aprendizaje afectivo. Además, las autorrevelaciones de los docentes por medio de clases online mejoró la satisfacción de los estudiantes sobre la relación profesor-alumno (Song, Kim y Luo, 2016; Song, Kim y Park, 2019).

Farani y Fatemi (2014) señalan que la autorrevelación docente mejora la predisposición del alumnado hacia el aprendizaje de la lengua inglesa ya que fomenta un clima distendido en la clase donde el alumnado siente que puede intercambiar sus experiencias con el profesorado y con sus compañeros de aula. De forma similar, Pishghadam y Askarzadeh (2009) descubrieron que la autorrevelación docente contribuyó a reducir la ansiedad del alumnado ante el aprendizaje de un nuevo idioma.

No obstante, Sidelinger, Frisby, McMullen y Heisler (2012) encontraron relaciones negativas entre la cantidad de autorrevelaciones docentes y el aprendizaje afectivo, de manera que cuando el profesorado reveló mucha información personal, los estudiantes mostraron menos afecto por el docente y por el curso. En esta línea, algunos estudios han determinado una asociación negativa entre la cantidad de autorrevelación docente y los comportamientos disruptivos del alumnado (Miller et al., 2014; Trad et al., 2012). Igualmente, Sidelinger, Nyeste, Madlock, Pollak y Wilkinson (2015) afirman que el alumnado presenta niveles más bajos de satisfacción con el curso cuando el profesorado revela información personal negativa.

\subsection{Motivación académica}

Brophy (1986) conceptualizó la motivación de los estudiantes como un rasgo y como un estado; la motivación como rasgo es una predisposición general y duradera hacia el aprendizaje, mientras que la motivación como estado es una actitud hacia una tarea o clase con un tema o profesor en particular, de forma que depende de la situación y es cambiante. Cayanus y Martin (2008) y Jebbour (2018) destacan una asociación positiva entre la autorrevelación docente y la motivación estado, de manera que cuando el colectivo docente comparte con sus estudiantes información personal de forma moderada, relevante y positiva, éstos están más motivados con la clase.

Mazer et al. (2007) y Saylag (2013) examinaron los efectos de la autorrevelación del docente en Facebook sobre la motivación de los estudiantes, encontrando que el alumnado que accedió al perfil de Facebook de un profesor con un alto grado de información personal mostró mayor motivación académica. Más concretamente, Aubry (2013) investigó los efectos de la autorrevelación del docente en Facebook sobre la motivación de los estudiantes, encontrando que, después de haber sido expuestos al perfil de Facebook del docente, los estudiantes experimentaron un cambio del tipo de motivación al pasar de estar externamente motivados a intrínsecamente motivados.

\subsection{Participación e interés de los estudiantes}

Ciertos estudios destacan la existencia de una correlación positiva entre la autorrevelación docente y la participación de los estudiantes en el aula (Goldstein y Benassi, 1994; Saylag, 2013). No obstante, Wambach y Brothen (1997) no encontraron una relación significativa entre la autorrevelación del docente y la participación de los estudiantes.

Cayanus, Martin y Myers (2008) descubrieron una asociación positiva entre la autorrevelación del docente y el uso de búsqueda de información por parte del alumnado, es decir, cuando los docentes comparten en el aula información privada, los estudiantes se sienten más libres para hacer preguntas y participar activamente en la búsqueda de información para comprender el contenido del curso. En el mismo sentido, Imlawi y Gregg (2014) hallaron una correlación positiva entre la autorrevelación docente a través de las redes sociales y la implicación de los estudiantes con el curso.

Asimismo, cuando las autorrevelaciones se realizan moderadamente, son relevantes y positivas, los estudiantes muestran mayor interés en el aula (Cayanus y Martin, 2004, 2008) y mayor compromiso en la clase (Borzea y Goodboy, 2016). 


\subsection{Comunicación profesor-alumno fuera del aula}

Según Fusani (1994), la comunicación profesor-alumno fuera del aula puede definirse como la interacción que tiene lugar entre el profesorado y el alumnado fuera de los horarios de clase fijados. Existe una correlación positiva entre la autorrevelación docente y la comunicación profesor-alumno fuera del aula, de forma que cuando el profesorado comparte información personal de manera moderada, relevante y positiva, los estudiantes interactúan más con ellos fuera de los horarios de clase establecidos (Baker et al., 2019; Cayanus y Martin, 2004; Singh, Gnanaselvam y Suppiah, 2018). Siguiendo esta línea, Cayanus et al. (2009) encontraron que la autorrevelación docente correlacionó de forma positiva con comunicarse con el docente para aprender más sobre cuestiones relacionadas con la asignatura y para demostrar que dominan el contenido de la materia, mientras que la negatividad de la autorrevelación se asoció negativamente con comunicarse con el docente para explicar por qué no se han realizado las tareas de clase y para causar una impresión favorable.

\subsection{Percepciones y evaluaciones sobre el docente}

La autorrevelación docente está relacionada con la evaluación que realiza el estudiante con respecto al profesorado (Sallinen-Kuparinen, 1992). Sorensen (1989) encontró que los docentes que emplearon autorrevelaciones positivas fueron evaluados positivamente mientras que los docentes que usaron autorrevelaciones negativas fueron evaluados negativamente. De manera similar, Lannutti y Strauman (2006) y Zhai (2012) hallaron que la autorrevelación que fue percibida como más honesta, positiva e intencional se asoció con evaluaciones más positivas del docente.

A través de las redes sociales, Sleigh, Smith y Laboe (2013) examinaron si un tipo específico de autorrevelación en el perfil de Facebook de un docente afectaría a las evaluaciones de los estudiantes sobre el profesor, creando para ello seis tipo de perfiles: políticamente conservador, políticamente liberal, social, profesional, religioso y familiar, encontrando que el profesor social fue percibido como menos hábil pero más popular aunque también su perfil fue percibido como inapropiado y entretenido, y que el alumnado reaccionó de forma más negativa a los perfiles políticos con que a los perfiles religiosos, familiares y profesionales.

Asimismo, la autorrevelación docente afecta a las percepciones del alumnado sobre la credibilidad del profesorado (McBride y Wahl, 2005). La credibilidad docente consiste en la percepción del estudiante sobre si el docente es creíble o no (McCroskey, 1992) y está compuesta por tres dimensiones: competencia, buena voluntad y confianza (McCroskey y Teven, 1999). Schrodt (2013) encontró que el profesorado que divulga información personal de manera moderada es más creíble. Myers et al. (2009) hallaron que los docentes que comparten información privada que es relevante para el alumnado o para el contenido de la clase, son más creíbles. Igualmente, Klebig, Goldonowicz, Mendes, Miller y Katt (2016) apuntan relaciones negativas entre la negatividad de las autorrevelaciones del profesorado y su credibilidad, concluyendo que cuando los docentes revelan información personal negativa, son percibidos por los estudiantes como menos creíbles.

En el contexto de las redes sociales, Mazer, Murphy y Simonds (2009) encontraron que el alumnado que accedió al Facebook de un profesor que presentaba mucha información personal le otorgó mayor credibilidad que el alumnado que observó el Facebook de un profesor que presentaba poca información privada. De la misma manera, Sarapin y Morris (2015) señalan que el profesorado que reveló más información privada en sus perfiles de Facebook fue percibido como más creíble que el profesorado que reveló escasa información. Por el contrario, Coffelt et al. (2014) apuntan que la credibilidad del profesorado dentro de la clase disminuye cuando aumenta la negatividad de la información privada de sus perfiles de Facebook. De forma similar, Wang, Novak, Scofield-Snow, Traylor y Zhou (2015) hallaron que el profesorado fue percibido como menos creíble cuando en sus perfiles de Facebook publicaron contenido relativo a problemas sentimentales y al consumo de alcohol.

Johnson (2011) analizó el efecto del contenido de los perfiles de Twitter del profesorado en su credibilidad, hallando que el perfil con contenido social fue percibido como más creíble que los perfiles con contenido profesional y mixto. Sin embargo, DeGroot, Young y VanSlette (2015) encontraron que el perfil profesional fue más creíble que el perfil social y que el perfil mixto y que el perfil mixto fue más creíble que el perfil social. En este sentido, Clark-Gordon y Goodboy (2019) destacaron que el perfil profesional fue el más competente mientras que el perfil social fue el que presentaba mayor buena voluntad.

Igualmente, Cayanus y Martin (2008) señalan que la autorrevelación docente correlaciona positivamente con las percepciones del alumnado sobre la claridad del docente, de manera que cuando éste revela información 
personal en el aula de forma moderada, relevante y positiva, los estudiantes perciben que el docente presenta el contenido de la asignatura de manera organizada y comprensible.

A modo de resumen, la Tabla 1 sintetiza las categorías identificadas, los artículos analizados y los resultados obtenidos.

\section{Tabla 1}

Síntesis de las categorías, artículos y resultados de la revisión

\begin{tabular}{|c|c|c|}
\hline Categorías & Artículos & Resultados \\
\hline Aprendizaje cognitivo & $\begin{array}{l}2 \text { (Gnanaselvam y Kepol (2018; } \\
\text { McCarthy y Schmeck, 1982). }\end{array}$ & $\begin{array}{c}\text { La autorrevelación docente } \\
\text { correlaciona positivamente con un } \\
\text { mayor recuerdo del material del } \\
\text { curso y con la mejora en la escritura } \\
\text { de los estudiantes. }\end{array}$ \\
\hline Aprendizaje afectivo & $\begin{array}{l}14 \text { (Cayanus y Martin, 2004, 2008; } \\
\text { Farani y Fatemi, 2014; Frisby et al., } \\
\text { 2012; Goodboy et al., 2014; Jebbour, } \\
\text { 2018; Mazer et al., 2007; Miller et al., } \\
\text { 2014; Pishghadam y Askarzadeh, } \\
\text { 2009; Sidelinger et al., 2015; Song et } \\
\text { al., 2016; Song et al., 2019; } \\
\text { Sorensen. 1989; Trad et al.. 2012). }\end{array}$ & $\begin{array}{c}\text { La autorrevelación docente se } \\
\text { relaciona positivamente con el } \\
\text { aprendizaje afectivo. Asimismo, la } \\
\text { cantidad y la negatividad de las } \\
\text { autorevelaciones afectan } \\
\text { negativamente al aprendizaje } \\
\text { afectivo. }\end{array}$ \\
\hline Motivación académica & $\begin{array}{c}5 \text { (Aubry, 2013; Cayanus y Martin, } \\
\text { 2008; Jebbour, 2018; Mazer et al., } \\
2007 \text { y Saylag, 2013). }\end{array}$ & $\begin{array}{c}\text { La autorrevelación docente se } \\
\text { relaciona positivamente con la } \\
\text { motivación académica de los } \\
\text { estudiantes. }\end{array}$ \\
\hline Participación e interés & $\begin{array}{c}8 \text { (Borzea y Goodboy, 2016; } \\
\text { Cayanus y Martin, 2004, 2008; } \\
\text { Cayanus et al., 2008; Goldstein y } \\
\text { Benassi, 1994; Imlawi y Gregg, 2014; } \\
\text { Saylag, 2013; Wambach y Brothen, } \\
\text { 1997). }\end{array}$ & $\begin{array}{c}\text { La autorrevelación docente se asocia } \\
\text { positivamente con la participación, la } \\
\text { implicación, el interés y el } \\
\text { compromiso de los estudiantes. }\end{array}$ \\
\hline $\begin{array}{l}\text { Comunicación profesor-alumno fuera } \\
\text { del aula }\end{array}$ & $\begin{array}{c}4 \text { (Baker et al., 2019; Cayanus y } \\
\text { Martin, 2004; Cayanus et al., 2009; } \\
\text { Singh et al., 2018). }\end{array}$ & $\begin{array}{c}\text { La autorrevelación docente } \\
\text { correlaciona positivamente con la } \\
\text { comunicación profesor-alumno fuera } \\
\text { del aula }\end{array}$ \\
\hline $\begin{array}{l}\text { Percepciones y evaluaciones sobre } \\
\text { el docente }\end{array}$ & $\begin{array}{l}15 \text { (Cayanus y Martin, 2008; Clark- } \\
\text { Gordon y Goodboy, 2019; Coffelt et } \\
\text { al., 2014; DeGroot et al., 2015; } \\
\text { Johnson, 2011; Klebig et al., 2016; } \\
\text { Lannutti y Strauman, 2006; Mazer et } \\
\text { al., 2009; Myers et al., 2009; Sarapin } \\
\text { y Morris, 2015; Schrodt, 2013; Sleigh } \\
\text { et al., 2013; Sorensen, 1989; Wang } \\
\text { et al., 2015; Zhai, 2012). }\end{array}$ & $\begin{array}{c}\text { La autorrevelación docente afecta a } \\
\text { las percepciones y evaluaciones de } \\
\text { los estudiantes sobre el docente. } \\
\text { Igualmente, impacta en la } \\
\text { credibilidad y en la claridad del } \\
\text { docente. }\end{array}$ \\
\hline
\end{tabular}

\section{Discusión y conclusiones}

El objetivo principal de esta investigación consistió en determinar el estado del arte sobre la autorrevelación docente mediante una revisión sistemática de la literatura, estableciendo su efecto en el proceso de enseñanza-aprendizaje.

Los resultados obtenidos en esta investigación destacan que la autorrevelación docente afecta al aprendizaje cognitivo, al aprendizaje afectivo, a la motivación académica, a la participación e interés, a la comunicación profesor-alumno fuera del aula y a las percepciones y evaluaciones sobre el docente. Por ello, es fundamental que los docentes sepan cómo pueden compartir información personal con sus estudiantes de manera efectiva y apropiada (Daly y Vangelisti, 2003).

Como indican Sidelinger et al. (2015), los docentes deben gestionar cómo compartir adecuadamente información personal con los estudiantes para crear un clima de aprendizaje enriquecedor. 
Los hallazgos obtenidos coinciden con diversos estudios que destacan que cuando los estudiantes evalúan positivamente el comportamiento de los docentes, influye de forma favorable en el proceso de enseñanzaaprendizaje (Baker y Goodboy, 2019; Frymier, Goldman y Claus, 2019; Violanti, Kelly, Garland y Christen, 2018) mientras que cuando evalúan negativamente la conducta del profesorado, afecta de manera desfavorable (Baker y Goodboy, 2018; Goodboy, Bolkan y Baker, 2018; Kelly, Romero, Morrow, Denton y Ducking, 2019). Como señalan McCroskey, Valencic y Richmond (2004), el comportamiento del profesorado constituye la fuente primaria de información mediante la cual los estudiantes perciben y evalúan a los docentes. Por ello, para que la docencia sea efectiva, los docentes deben comportarse adecuadamente (Gray, Anderman y O'Connell, 2011).

La importancia de las autorrevelaciones de los docentes en el proceso de enseñanza-aprendizaje, se ha comprobado en Estados Unidos, encontrándose solo algunas investigaciones realizadas en otros países como Irán (Farani y Fatemi, 2014; Pishghadam y Askarzadeh, 2009), Malasia (Gnanaselvam y Kepol, 2018; Singh et al., 2018), Marruecos (Jebbour, 2018), Turquía (Saylag, 2013) y Corea del Sur (Song et al., 2019). Como apuntan Cayanus y Martin (2016), la investigación sobre autorrevelación docente se ha limitado fundamentalmente al entorno de la universidad en los Estados Unidos. Por ello, se propone el desarrollo de estudios transculturales sobre autorrevelación docente para determinar si los resultados obtenidos en otros países coinciden o difieren con los hallados disponibles. Siguiendo esta línea, se sugiere como investigación futura el análisis de las propiedades psicométricas de la Escala de Autorrevelación Docente (Cayanus y Martin, 2008) con muestras de otros países y culturas debido a la ausencia de adaptaciones y validaciones de dicha escala en otros idiomas. Como concluye Waldeck (2011), pese a la riqueza del trabajo existente sobre autorrevelación docente, nuestra comprensión de cómo la cultura afecta a las autorrevelaciones y las percepciones de los estudiantes sobre las mismas es limitada.

Igualmente, como se aprecia en este estudio, la información personal que los docentes revelan y comparten a través de sus perfiles en las redes sociales afecta al proceso de enseñanza-aprendizaje, por lo que en este sentido se proponen estudios sobre el impacto de las autorrevelaciones de los docentes en las redes sociales sobre otras variables del proceso de enseñanza-aprendizaje como el interés, la satisfacción y el empoderamiento del estudiante. Además, se sugieren investigaciones que analicen el efecto de las autorrevelaciones docentes en las redes sociales sobre la perdurabilidad de variables asociadas al proceso de enseñanza-aprendizaje como la motivación académica del estudiante a lo largo del curso o si ésta se ve alterada tras observar la información personal que los docentes comparten en sus perfiles online. Como indican Cayanus y Martin (2016) al respecto, la proliferación del uso de las redes sociales por parte de los docentes les da un contexto completamente diferente dentro del cual pueden decidir la información privada que desean compartir con el resto de usuarios, pudiendo los estudiantes responder de manera diferente en comparación con las revelaciones personales que realiza el profesorado en clase. En definitiva, los docentes deben gestionar la información personal que publican y comparten en las redes sociales ya que afecta a las percepciones y evaluaciones del alumnado sobre el docente y, por consiguiente, al proceso de enseñanzaaprendizaje (Mikulec, 2012).

Como limitaciones del estudio destacamos la ausencia de investigaciones redactadas en otras lenguas a las que no hemos podido tener acceso, de manera que no se puede asumir tener todos los datos existentes relativos a la temática abordada. Sin embargo, a pesar de esta limitación, la presente investigación sugiere importantes implicaciones prácticas, ya que los docentes que comparten o tienen intención de compartir con sus alumnos información de carácter personal, deben hacerlo de forma moderada y debe ser relevante con el contenido del curso y positiva para que tenga un efecto favorable sobre el proceso de enseñanzaaprendizaje. En definitiva, esta revisión permite a los docentes conocer el modo en el que deben revelar y compartir información personal con los estudiantes para que tenga un efecto favorable sobre el proceso de enseñanza-aprendizaje, suponiendo asimismo un punto de partida para nuevos enfoques teóricos.

Atendiendo al presente análisis, las autorrevelaciones realizadas por los docentes tanto dentro del aula como en las redes sociales constituyen un elemento fundamental para el proceso de enseñanza-aprendizaje. Como señalan Mazer et al. (2007), la autorrevelación docente supone una decisión estratégica con implicaciones tanto para la relación profesor-alumno como para el éxito de la clase. Por ello, aprender a utilizar esta herramienta pedagógica de forma adecuada permitirá a los docentes aumentar la efectividad de su docencia (Kaufmann y Lane, 2014). Cuando se usa apropiadamente, la autorrevelación docente ayuda a crear un ambiente de clase positivo y a construir relaciones satisfactorias entre el profesorado y el alumnado, mientras que cuando se emplea de manera inadecuada, resulta perjudicial para el clima del aula (Cayanus y Martin, 2016). 
Así, Rasmussen y Mishna (2008) establecen ciertas directrices para el uso de las autorrevelaciones por parte de los docentes en al aula: (a) la autorrevelación debe estar siempre al servicio del aprendizaje de los estudiantes; (b) los docentes deben tener en cuenta que el impacto de las autorrevelaciones dependerá, en parte, de las diferencias de poder existentes entre ambos; (c) el colectivo docente debe reflexionar sobre los motivos por los que revelan y comparten información personal con los estudiantes; (d) el contenido de las autorrevelaciones debe situarse dentro de los límites razonables de intimidad y (e) el profesorado puede considerar preguntar a los estudiantes por sus reacciones ante las autorrevelaciones realizadas. En síntesis, como concluyen Hosek y Presley (2018), las autorrevelaciones del profesorado deben seguir en todo momento tres principios fundamentales: (a) que se relacionen con el contenido del curso y el material de clase; (b) que sean positivas y (c) que fomenten el desarrollo de relaciones satisfactorias entre el profesorado y el alumnado.

\section{Referencias}

Aubry, J. (2013). Facebook-Induced Motivation Shifts in a French Online Course.

TechTrends, 57(6), 81-87. https://doi.org/10.1007/s11528-013-0705-6

Baker, J. P., Clark-Gordon, C. V. y Myers, S. A. (2019). Using emotional response theory to examine dramatic teaching behaviors and student approach-avoidance behaviors. Communication Education, 68(2), 193-214. https://doi.org/10.1080/10510974.2016.1212912

Baker, J. P. y Goodboy, A. K. (2018). Students' self-determination as a consequence of instructor misbehaviors. Communication Research Reports, 35(1), 68-73. https://doi.org/10.1080/08824096.2017.1366305

Baker, J. P. y Goodboy, A. K. (2019). The choice is yours: the effects of autonomy-supportive instruction on students' learning and communication. Communication Education, 68(1), 80-102. https://doi.org/10.1080/03634523.2018.1536793

Bloom, B. S. (1976). Human characteristics and school learning. New York: McGraw-Hill.

Borzea, D. y Goodboy, A. K. (2016). When instructors self-disclose but misbehave: Conditional effects on student engagement and interest. Communication Studies, 67(5), 548-566. https://doi.org/10.1080/10510974.2016.1212912

Braun, V. y Clarke, V. (2006). Using thematic analysis in psychology. Qualitative Research in Psychology, 3(2), 77-101. https://doi.org/10.1191/1478088706qp063oa

Brophy, J. (1986). Socializing students' motivation to learn. East Lansing, Michigan: Michigan State University Press. Buskist, W. y Saville, B. (2004). Rapport-building: Creating positive emotional contexts for enhancing teaching and learning. En B. Perlman, L. I. McCann y S. H. McFadden's (Eds.), Lessons learned: Practical advice for the teaching of psychology (Vol. 2, pp. 149-155). Washington, DC: American Psychological Society.

Cayanus, J. L. (2004). Effective Instructional Practice: Using Teacher Self-Disclosure as an Instructional Tool.

Communication Teacher, 18(1), 6-9. https://doi.org/10.1080/1740462032000142095

Cayanus, J. L. y Martin, M. M. (2004). An instructor self-disclosure scale. Communication Research Reports, 21(3), 252263. https://doi.org/10.1080/08824090409359987

Cayanus, J. L. y Martin, M. M. (2008). Teacher self-disclosure: Amount, relevance, and negativity. Communication Quarterly, 56(3), 325-341. https://doi.org/10.1080/01463370802241492

Cayanus, J. y Martin, M. M. (2016). Teacher Self-Disclosure. En P. L. Witt (Ed.), Communication and Learning (pp. 241258). Boston: De Gruyter. https://doi.org/10.1515/9781501502446-011

Cayanus, J. L., Martin, M. M. y Goodboy, A. K. (2009). The relation between teacher self-disclosure and student motives to communicate. Communication Research Reports, 26(2), 105-113. https://doi.org/10.1080/08824090902861523

Cayanus, J. L., Martin, M. M. y Myers, S. A. (2008). The relationship between perceived instructor self-disclosure and college student information seeking. Texas Speech Communication Journal, 33(1), 20-27.

Clark-Gordon, C. V. y Goodboy, A. K. (2019). Instructor Self-Disclosure and Third-Party Generated Warrants: Student Perceptions of Professor Social Media Use. Western Journal of Communication, 1-19. https://doi.org/10.1080/10570314.2019.1649453

Coffelt, T. A., Strayhorn, J. y Tillson, L. D. (2014). Perceptions of Teachers' Disclosures on Facebook and their Impact on Credibility. Kentucky Journal of Communication, 33(2), 25-43.

Cooper, P. J. y Simonds, C. (1999). Communication for the classroom teacher. Boston, Massachusetts: Allyn Bacon.

Cozby, P. C. (1973). Self-disclosure: a literature review. Psychological Review, 79(2), 73-91. https://doi.org/10.1037/h0033950

Dalto, C. A., Ajzen, I. y Kaplan, K. J. (1979). Self-disclosure and attraction: Effects of intimacy and desirability on beliefs and attitudes. Journal of Research in Personality, 13(2), 127-138. https://doi.org/10.1016/0092-6566(79)90025-4

Daly, J. A. y Vangelisti, A. L. (2003). Skillfully instructing learners: How communicators effectively convey messages. En J. O. Greene y B. R. Burleson (Eds.), Handbook of communication and social interaction skills (pp. 871-908). New York: Routledge.

DeGroot, J. M., Young, V. J. y VanSlette, S. H. (2015). Twitter use and its Effects on Student perception of Instructor Credibility. Communication Education, 64(4), 419-437. https://doi.org/10.1080/03634523.2015.1014386 
Deiro, J. (2005). Teachers do make a difference: The teacher's guide to connecting with students. Thousand Oaks, California: Corwin Press.

DiVerniero, R. A. y Hosek, A. M. (2011). Students' Perceptions and Communicative Management of Instructors' Online Self-Disclosure. Communication Quarterly, 59(4), 428-449. https://doi.org/10.1080/01463373.2011.597275

Downs, V. C., Javidi, M. y Nussbaum, J. F. (1988). An analysis of teacher's verbal communication within the college classroom: Use of humor, self-disclosure, and narratives. Communication Education, 37(2), 127-141.

https://doi.org/10.1080/03634528809378710

Edwards, A., Edwards, C., Shaver, C. y Oaks, M. (2009). Computer-mediated word-of-mouth communication on ratemyprofessors.com: Expectancy effects on student cognitive and behavioral learning. Journal of Computer-Mediated Communication, 14(2), 368-392. https://doi.org/10.1111/i.1083-6101.2009.01445.x

Farani, S. T. y Fatemi, A. H. (2014). The Impact of Teacher's Self-disclosure on Students' Attitude towards Language Learning in a Foreign Language Context. Theory and Practice in Language Studies, 4(11), 2415-2422.

https://doi.org/10.4304/tpls.4.11.2415-2422

Frisby, B. N. y Sidelinger, R. J. (2013). Violating student expectations: Student disclosures and student reactions in the college classroom. Communication Studies, 64, 241-258. https://doi.org/10.1080/10510974.2012.755636

Frymier, A. B., Goldman, Z. W. y Claus, C. J. (2019). Why nonverbal immediacy matters: A motivation explanation. Communication Quarterly, 67(5), 526-539. https://doi.org/10.1080/01463373.2019.1668442

Frymier, A. B. y Houser, M. L. (2000). The teacher-student relationship as an interpersonal relationship. Communication Education, 49(3), 207-219. https://doi.org/10.1080/03634520009379209

Fusani, D. S. (1994). "Extra-class" communication: Frequency, immediacy, self-disclosure, and satisfaction in studentfaculty interaction outside of the classroom. Journal of Applied Communication Research, 22(3), 232-255. https://doi.org/10.1080/00909889409365400

Gnanaselvam, P. y Kepol, N. (2018). Perceived Teachers' Self-disclosure, Writing Performance and Gender of Malaysian ESL Undergraduates. Pertanika Journal of Social Sciences \& Humanities, 26(4), 2195-2210.

Goldstein, G. S. y Benassi, V. A. (1994). The relation between teacher self-disclosure and student classroom participation. Teaching of Psychology, 21(4), 212-216. https://doi.org/10.1207/s15328023top2104 2

Goodboy, A. K., Bolkan, S. y Baker, J. P. (2018). Instructor misbehaviors impede students' cognitive learning: testing the causal assumption. Communication Education, 67(3), 308-329. https://doi.org/10.1080/03634523.2018.1465192 Goodboy, A. K., Carton, S. T., Goldman, Z. W., Gozanski, T. A., Tyler, W. J. y Johnson, N. R. (2014). Discouraging instructional dissent and facilitating students' learning experiences through instructor self-disclosure. Southern Communication Journal, 79(2), 114-129. https://doi.org/10.1080/1041794x.2013.865256

Gray, D. L., Anderman, E. M. y O'Connell, A. A. (2011). Associations of teacher credibility and teacher affinity with learning outcomes in health classrooms. Social Psychology of Education, 14(2), 185-208.

https://doi.org/10.1007/s11218-010-9143-x

Guest, G., MacQueen, K. M. y Namey, E. E. (2012). Applied Thematic Analysis. Thousand Oaks, CA: Sage Publications. https://doi.org/10.4135/9781483384436

Hosek, A. M. y Presley, R. (2018). College Student Perceptions of The (In) Appropriateness and Functions of Teacher Disclosure. College Teaching, 66(2), 63-72. https://doi.org/10.1080/87567555.2017.1385587

Hosek, A. M. y Thompson, J. (2009). Communication Privacy Management and College Instruction: Exploring the Rules and Boundaries that Frame Instructor Private Disclosures. Communication Education, 58(3), 327-349.

https://doi.org/10.1080/03634520902777585

Imlawi, J. y Gregg, D. (2014). Engagement in online social networks: The impact of self-disclosure and humor. International Journal of Human-Computer Interaction, 30(2), 106-125. https://doi.org/10.1080/10447318.2013.839901 Javidi, M. N. y Long, L. W. (1989). Teachers' use of humor, self-disclosure, and narrative activity as a function of experience. Communication Research Reports, 6(1), 47-52. https://doi.org/10.1080/08824098909359831

Jebbour, M. (2018). University Students' Perceptions of the Effects of Teacher Self-Disclosure in the English Language Classroom. Journal of English Language Teaching and Linguistics, 3(3), 275-285. https://doi.org/10.21462/jeltl.v3i3.166 Johnson, K. A. (2011). The effect of Twitter posts on students' perceptions of instructor credibility. Learning, Media and Technology, 36(1), 21-38. https://doi.org/10.1080/17439884.2010.534798

Kaufmann, R. M. y Lane, D. (2014). Examining communication privacy management in the middle school classroom:

Perceived gains and consequences. Educational Research, 56(1), 13-27. https://doi.org/10.1080/00131881.2013.874145 Kearney, P., Plax, T. G., Hays, E. R. e Ivey, M. J. (1991). College teacher misbehaviors: What students don't like about what teachers say or do. Communication Quarterly, 39(4), 309-324. https://doi.org/10.1080/01463379109369808 Kelly, S., Romero, A., Morrow, J. A., Denton, Z. y Ducking, J. (2019). Instructor misbehaviors and math anxiety. Communication Reports, 1-14. https://doi.org/10.1080/08934215.2019.1675737

Klebig, B., Goldonowicz, J., Mendes, E., Miller, A. N. y Katt, J. (2016). The Combined Effects of Instructor Communicative Behaviors, Instructor Credibility and Student Personality Traits on Incivility in the College Classroom. Communication Research Reports, 33(2), 152-158. https://doi.org/10.1080/08824096.2016.1154837

Lannutti, P. J. y Strauman, E. C. (2006). Classroom communication: The influence of instructor self-disclosure on student evaluations. Communication Quarterly, 54(1), 89-99. https://doi.org/10.1080/01463370500270496 
Mazer, J. P., Murphy, R. E. y Simonds, C. J. (2007). I'll see you on "Facebook": The effects of computer-mediated teacher self-disclosure on student motivation, affective learning, and classroom climate. Communication Education, 56(1), 1-17. https://doi.org/10.1080/03634520601009710

Mazer, J. P., Murphy, R. E. y Simonds, C. J. (2009). The Effects of Teacher Self-disclosure via Facebook on Teacher Credibility. Learning, Media and Technology, 34(2), 175-183. https://doi.org/10.1080/17439880902923655

McBride, M. y Wahl, S. (2005). 'To say or not to say:' Teachers' management of privacy boundaries in the classroom. Texas Speech Communication Journal, 30, 8-22.

McCarthy, P. R. y Schmeck, R. R. (1982). Effects of teacher self-disclosure on student learning and perceptions of teacher. College Student Journal, 16(1), 45-49.

McCroskey, J. C. (1992). An introduction to communication in the classroom. Edina, Minnesota: Burgess International. McCroskey, J. C. y Teven, J. J. (1999). Goodwill: A reexamination of the construct and its measurement. Communication Monographs, 66(1), 90-103. https://doi.org/10.1080/03637759909376464

McCroskey, J. C., Valencic, K. M. y Richmond, V. P. (2004). Toward a general model of instructional communication. Communication Quarterly, 52(3), 197-210. https://doi.org/10.1080/01463370409370192

Meluch, A., Feehan, K. y Starcher, S. (2019). Instructor Disclosures of Communication Apprehension and Student Perceptions of Instructor Credibility in the Public Speaking Classroom. International Journal of Teaching and Learning in Higher Education, 31(2), 299-309.

Mikulec, E. A. (2012). Professional Faces: Pre-service Secondary Teachers' Awareness of Issues of Self-disclosure on Social-networking Sites. Current Issues in Education, 15(3), 1-16.

Miller, A. N., Katt, J. A., Brown, T. y Sivo, S. A. (2014). The relationship of instructor self-disclosure, nonverbal immediacy, and credibility to student incivility in the college classroom. Communication Education, 63(1), 1-16. https://doi.org/10.1080/03634523.2013.835054

Myers, S. A., Brann, M. y Members of Comm 600. (2009). College Students' Perceptions of How Instructors Establish and Enhance Credibility Through Self-Disclosure. Qualitative Research Reports in Communication, 10(1), 9-16.

Nussbaum, J. F., Comadena, M. E. y Holladay, S. J. (1987). Classroom verbal behaviors of highly effective teachers. Journal of Thought, 22, 73-80.

Nussbaum, J. F. y Scott, M. D. (1979). Instructor communication behaviors and their relationship to classroom learning. Annals of the International Communication Association, 3(1), 561-583.

https://doi.org/10.1080/23808985.1979.11923783

Paluckaite, U. y Zardeckaite-Matulaitiene, K. (2015). The appropriateness of the relevant and irrelevant teacher's selfdisclosure: Students' and their teachers' attitudes. European Scientific Journal, 11, 18-35.

Pishghadam, R. y Askarzadeh, R. (2009). The impacts of teacher self-disclosure on the speaking ability of EFL learners. Iranian EFL Journal, 3, 37-46.

Rahimi, A. y Bigdeli, R. A. (2016). Iranian EFL Teachers' Perceptions of Teacher Self-Disclosure. Iranian Journal of Language Teaching Research, 4(1), 83-96.

Rasmussen, B. M. y Mishna, F. (2008). A fine balance: Instructor self-disclosure in the classroom. Journal of Teaching in Social Work, 28(1-2), 191-207. https://doi.org/10.1080/08841230802179274

Sallinen-Kuparinen, A. (1992). Teacher communicator style. Communication Education, 41(2), 153-166.

https://doi.org/10.1080/03634529209378877

Sarapin, S. H. y Morris, P. L. (2015). Faculty and Facebook friending: Instructor-student online social communication from the professor's perspective. The Internet and Higher Education, 27, 14-23.

https://doi.org/10.1016/j.iheduc.2015.04.001

Saylag, R. (2013). Facebook as a Tool in Fostering EFL Teachers' Establishment of Interpersonal Relations with Students Through Self-disclosure. Procedia - Social and Behavioral Sciences, 82, 680-685.

https://doi.org/10.1016/i.sbspro.2013.06.329

Schrodt, P. (2013). Content relevance and students' comfort with disclosure as moderators of instructor disclosures and credibility in the college classroom. Communication Education, 62(4), 352-375.

https://doi.org/10.1080/03634523.2013.807348

Sidelinger, R. J., Frisby, B. N., McMullen, A. L. y Heisler, J. (2012). Developing student-to-student connectedness: An examination of instructors' humor, nonverbal immediacy, and self-disclosure in public speaking courses. Basic Communication Course Annual, 24, 81-121.

Sidelinger, R. J., Nyeste, M. C., Madlock, P. E., Pollak, J. y Wilkinson, J. (2015). Instructor privacy management in the classroom: Exploring instructors' ineffective communication and student communication satisfaction. Communication Studies, 66(5), 569-589. https://doi.org/10.1080/10510974.2015.1034875

Singh, A., Gnanaselvam, P. y Suppiah, S. M. (2018). The relationship between perceived teachers' self-disclosure and out-of-classroom communication among Malaysian undergraduates in a private institution of higher learning. Asian Journal of University Education, 14(1), 37-57.

Sleigh, M. J., Smith, A. W. y Laboe, J. (2013). Professors' Facebook Content Affects Students' Perceptions and Expectations. Cyberpsychology, Behavior and Social Networking, 16(7), 489-496.

https://doi.org/10.1089/cyber.2012.0561

Song, H., Kim, J. y Luo, W. (2016). Teacher-student relationships in online classes: A role of teacher self-disclosure. Computers in Human Behavior, 54, 436-443. https://doi.org/10.1016/j.chb.2015.07.037 
Song, H., Kim, J. y Park, N. (2019). I Know My Professor: Teacher Self-Disclosure in Online Education and a Mediating Role of Social Presence. International Journal of Human-Computer Interaction, 35(6), 448-455.

https://doi.org/10.1080/10447318.2018.1455126

Sorensen, G. (1989). The relationship among teachers' self-disclosive statements, students' perceptions, and affective learning. Communication Education, 38(3), 259-276. https://doi.org/10.1080/03634528909378762

Sumuer, E., Esfer, S. y Yildirim, S. (2014). Teachers' Facebook use: their use habits, intensity, self-disclosure, privacy settings, and activities on Facebook. Educational Studies, 40(5), 537-553.

https://doi.org/10.1080/03055698.2014.952713

Trad, L., Baker, N., Blackman, H., Glynn, K., Wright, A. y Miller, A. N. (2012). Student Incivility and Instructor Communication in the College Classroom. Florida Communication Journal, 40(1), 47-53.

Violanti, M. T., Kelly, S. E., Garland, M. E. y Christen, S. (2018). Instructor clarity, humor, immediacy, and student learning: Replication and extension. Communication Studies, 69(3), 251-262.

https://doi.org/10.1080/10510974.2018.1466718

Waldeck, J. H. (2011). Teacher Self-Disclosure. En W. Donsbach (Ed.), The International Encyclopedia of

Communication (pp.1-3). New Jersey: John Wiley \& Sons. https://doi.org/10.1002/9781405186407.wbiect015.pub2

Wambach, C. y Brothen, T. (1997). Teacher self-disclosure and student classroom participation revisited. Teaching of Psychology, 24(4), 262-263. https://doi.org/10.1207/s15328023top2404 7

Wang, Z.; Novak, H.; Scofield-Snow, H.; Traylor, S. y Zhou, Y. (2015). Am I Disclosing Too Much? Student Perceptions of Teacher Credibility via Facebook. The Journal of Social Media in Society, 4(1), 5-37.

Webb, N. G. (2014). To Share or not to share: GTA self-disclosure in the college classroom. The Official Journal of the Georgia Communication Association, 83, 7-10.

Zardeckaite-Matulaitiene, K. y Paluckaite, U. (2013). The relation between teacher's self-disclosure and student's motivation to learn. European Scientific Journal, 9(28), 456-469. https://dx.doi.org/10.19044/esj.2013.v9n28p\%25p Zhai, L. (2012). The influence of instructor self-disclosure on students' evaluations: an intercultural comparison. International Journal of Applied Science and Technology, 2(3), 58-66.

Zhang, S. (2010). Secondary preservice teachers' perspectives on teacher self-disclosure on citizenship curriculum. Action in Teacher Education, 32(2), 82-96. https://doi.org/10.1080/01626620.2010.10463552

Zhang, S., Shi, Q., Tonelson, S. y Robinson, J. (2009). Preservice and Inservice Teachers' Perceptions of Appropriateness of Teacher Self-Disclosure. Teaching and Teacher Education, 25(8), 1117-1124.

https://doi.org/10.1016/.tate.2008.07.011 
Anexo 1.

Artículos incluidos en la revisión.

\begin{tabular}{|c|c|c|c|c|}
\hline Rango & Autores & Año & Nombre de la revista & Título del artículo \\
\hline 1 & Aubry & 2013 & TechTrends & Facebook-Induced Motivation Shifts in a French Online Course. \\
\hline 2 & Baker et al. & 2019 & Communication Education & $\begin{array}{l}\text { Using emotional response theory to examine dramatic teaching } \\
\text { behaviors and student approach-avoidance behaviors. }\end{array}$ \\
\hline 3 & $\begin{array}{l}\text { Borzea y } \\
\text { Goodboy }\end{array}$ & 2016 & Communication Studies & $\begin{array}{c}\text { When instructors self-disclose but misbehave: Conditional effects on } \\
\text { student engagement and interest. }\end{array}$ \\
\hline 4 & Cayanus y Martin & 2004 & $\begin{array}{l}\text { Communication Research } \\
\text { Reports }\end{array}$ & An instructor self-disclosure scale. \\
\hline 5 & Cayanus y Martin & 2008 & Communication Quarterly & Teacher self-disclosure: Amount, relevance, and negativity. \\
\hline 6 & Cayanus et al. & 2008 & $\begin{array}{l}\text { Texas Speech Communication } \\
\text { Journal }\end{array}$ & $\begin{array}{l}\text { The relationship between perceived instructor self-disclosure and } \\
\text { college student information seeking. }\end{array}$ \\
\hline 7 & Cayanus et al. & 2009 & $\begin{array}{l}\text { Communication Research } \\
\text { Reports }\end{array}$ & $\begin{array}{l}\text { The relation between teacher self-disclosure and student motives to } \\
\text { communicate. }\end{array}$ \\
\hline 8 & $\begin{array}{l}\text { Clark-Gordon y } \\
\text { Goodboy }\end{array}$ & 2019 & $\begin{array}{l}\text { Western Journal of } \\
\text { Communication }\end{array}$ & $\begin{array}{l}\text { Instructor Self-Disclosure and Third-Party Generated Warrants: } \\
\text { Student Perceptions of Professor Social Media Use. }\end{array}$ \\
\hline 9 & Coffelt et al. & 2014 & $\begin{array}{l}\text { Kentucky Journal of } \\
\text { Communication }\end{array}$ & $\begin{array}{c}\text { Perceptions of Teachers' Disclosures on Facebook and their Impact } \\
\text { on Credibility. }\end{array}$ \\
\hline 10 & DeGroot et al. & 2015 & Communication Education & $\begin{array}{c}\text { Twitter use and its Effects on Student perception of Instructor } \\
\text { Credibility. }\end{array}$ \\
\hline 11 & Farani y Fatemi & 2014 & $\begin{array}{l}\text { Theory and Practice in } \\
\text { Language Studies }\end{array}$ & $\begin{array}{l}\text { The Impact of Teacher's Self-disclosure on Students' Attitude } \\
\text { towards Language Learning in a Foreign Language Context. }\end{array}$ \\
\hline 12 & $\begin{array}{l}\text { Gnanaselvam y } \\
\text { Kepol }\end{array}$ & 2018 & $\begin{array}{l}\text { Pertanika Journal of Social } \\
\text { Sciences \& Humanities }\end{array}$ & $\begin{array}{c}\text { Perceived Teachers' Self-disclosure, Writing Performance and } \\
\text { Gender of Malaysian ESL Undergraduates. }\end{array}$ \\
\hline 13 & $\begin{array}{l}\text { Goldstein y } \\
\text { Benassi }\end{array}$ & 1994 & Teaching of Psychology & $\begin{array}{c}\text { The relation between teacher self-disclosure and student classroom } \\
\text { participation. }\end{array}$ \\
\hline 14 & Goodboy et al. & 2014 & $\begin{array}{l}\text { Southern Communication } \\
\text { Journal }\end{array}$ & $\begin{array}{c}\text { Discouraging instructional dissent and facilitating students' learning } \\
\text { experiences through instructor self-disclosure. }\end{array}$ \\
\hline 15 & Imlawi y Gregg & 2014 & $\begin{array}{l}\text { International Journal of } \\
\text { Human-Computer Interaction }\end{array}$ & $\begin{array}{l}\text { Engagement in online social networks: The impact of self-disclosure } \\
\text { and humor. }\end{array}$ \\
\hline
\end{tabular}


Johnson

Mazer et al.

Myers et al.

Pishghadam y

Askarzadeh

Sarapin y Morris

Schrodt
Basic Communication Course Annual

Journal of English Language Teaching and Linguistics Learning, Media and

Technology

Communication Research Reports

Communication Quarterly

Communication Education

\section{Learning, Media and} Technology

College Student Journal

Communication Education

2009 Qualitative Research Reports in Communication Iranian EFL Journal

\section{The Internet and Higher} Education

Procedia - Social and

Behavioral Sciences

Communication Education

$$
\text { Communication Studies }
$$

University Students' Perceptions of the Effects of Teacher SelfDisclosure in the English Language Classroom.

The effect of Twitter posts on students' perceptions of instructor credibility.

The Combined Effects of Instructor Communicative Behaviors, Instructor Credibility and Student Personality Traits on Incivility in the College Classroom.

Classroom communication: The influence of instructor self-disclosure on student evaluations.

I'll see you on "Facebook": The effects of computer-mediated teacher self-disclosure on student motivation, affective learning, and classroom climate.

The Effects of Teacher Self-disclosure via Facebook on Teacher Credibility.

Effects of teacher self-disclosure on student learning and perceptions of teacher.

The relationship of instructor self-disclosure, nonverbal immediacy, and credibility to student incivility in the college classroom.

College Students' Perceptions of How Instructors Establish and Enhance Credibility Through Self-Disclosure.

The impacts of teacher self-disclosure on the speaking ability of EFL learners.

Faculty and Facebook friending: Instructor-student online social communication from the professor's perspective.

Facebook as a Tool in Fostering EFL Teachers' Establishment of Interpersonal Relations with Students Through Self-disclosure.

Content relevance and students' comfort with disclosure as moderators of instructor disclosures and credibility in the college classroom.

Developing student-to-student connectedness: An examination of instructors' humor, nonverbal immediacy, and self-disclosure in public speaking courses.

Instructor privacy management in the classroom: Exploring instructors' ineffective communication and student communication satisfaction. 
Singh et al.

2018

Asian Journal of University Education

Sleigh et al.

2013

33

Song et al.

2016

Cyberpsychology, Behavior and Social Networking Computers in Human

$$
\text { Behavior }
$$

34

Song et al.

Sorensen

2019

International Journal of

Human-Computer Interaction

1989 Communication Education

36

37

Trad et al.

2012

Wambach y

Brothen

38

Wang et al.

39
Zhai
1997

2015

2012

\section{Communication Education}

Florida Communication

$$
\text { Journal }
$$

Teaching of Psychology

$$
\begin{gathered}
\text { The Journal of Social Media in } \\
\text { Society }
\end{gathered}
$$

International Journal of Applied Science and Technology
The relationship between perceived teachers' self-disclosure and out-of-classroom communication among Malaysian undergraduates in a private institution of higher learning.

Professors' Facebook Content Affects Students' Perceptions and Expectations.

Teacher-student relationships in online classes: A role of teacher self-disclosure.

I Know My Professor: Teacher Self-Disclosure in Online Education and a Mediating Role of Social Presence.

The relationship among teachers' self-disclosive statements, students' perceptions, and affective learning.

Student Incivility and Instructor Communication in the College Classroom.

Teacher self-disclosure and student classroom participation revisited.

Am I Disclosing Too Much? Student Perceptions of Teacher Credibility via Facebook.

The influence of instructor self-disclosure on students' evaluations: an intercultural comparison. 